\title{
Comparative approaches to same/different abstract-concept learning
}

\author{
A. A. Wright $^{1}$ - D. M. Kelly ${ }^{2}$
}

Published online: 14 April 2017

(C) Psychonomic Society, Inc. 2017

Summary Martinho and Kacelnik (2016) imprinted newly hatched ducklings (Anas platyrhynchos domestica) with a moving pair of either same or different objects, and following only one session, the ducklings accurately transferred the samel different relationship to novel object pairs that maintained the training relationship. This rapid learning and transfer of the concepts same and different far outstrips the more gradual learning of these basic concepts by animals in associative-learning tasks in which reinforcement is given for correct responses.

Keywords Concept learning · Comparative cognition

In the past 20 or so years, there have been many examples of remarkable intelligent behavior by (nonhuman) animals. Some of the most remarkable have come from birds, including species of the corvidae family: tool making and tool use by New Caledonian crows (Corvus moneduloides); magpies and nutcrackers (Pica pica and Nucifraga columbiana) identifying their "selfs" in mirrors; and nutcrackers storing thousands of pine seeds recovered months later when covered by snow (see Wright et al., 2017, for further details). These examples of remarkable intelligent behaviors are but a few of the sorts that have long been considered unique to humans. Although truly remarkable, these feats of intelligence are not directly comparable. One approach was to develop a task that all species to be compared could readily perform, and one that involved higherorder abstraction with a functional manipulation leading to successful concept learning (Wright \& Katz, 2006). The task that we chose was the sameldifferent task, with the functional manipulation being systematic expansion of the number of exemplars leading to successful concept learning. Monkeys (rhesus and capuchin) and pigeons were trained with pairs of pictures

D. M. Kelly

debbie.kelly@umanitoba.ca

1 University of Texas Health Science Center, Houston, TX, USA

2 University of Manitoba, Winnipeg, Manitoba, Canada (same, different), beginning with a small set of eight pictures (64 exemplars of the same/different rule), followed by transfer tests with novel stimuli for the assessment of concept learning. The cycle of doubling the training set, training with the doubled set, and transfer testing was repeated multiple times, leading to the result that transfer (concept learning) was eventually equivalent to the baseline training performance, a measure of full concept learning. Concept learning progressively increased from chance performance $(50 \%)$ and equaled the training performance (80\%-85\% correct) with a set size of 128 pictures for monkeys and 256 pictures for pigeons. Although these species had learned the original eight-picture set at about the same rate (3,000-4,000 trials), all species required fewer trials to learn the subsequent expanded training sets. These are reasonably proficient learning rates by any associative-learning standard, but nevertheless they are many orders of magnitude longer than the near-instantaneous imprinting learning shown by the newborn ducklings in Martinho and Kacelnik's (2016) study.

The study by Martinho and Kacelnik (2016) used a very powerful and ecologically relevant form of learning, imprinting, to approach the study of avian concept learning from a new direction. Imprinting behavior is a combination of hard-wired instinctual behavior and more flexible associative learning in which ducklings learn to follow a moving stimulus (a conspecific, another animal including humans, or moving objects) during a sensitive period of 24-48 h after hatching. Konrad Lorenz observed that such animals have an inner drive to carry out instinctive behaviors, and if they do not encounter the right stimulus, they will eventually engage in the behavior with an inappropriate stimulus, such as the moving objects used by Martinho and Kacelnik. In the Martinho and Kacelnik study, newly hatched domesticated ducklings (Anas platyrhynchos domestica) were exposed to pairs of three-dimensional objects, suspended at eye level, and moved around the perimeter of a circular arena for 25 $\mathrm{min}$. One group of ducklings was exposed in that manner to two identical objects, whereas another group was exposed to two different objects. These researchers were able to use this exposure procedure to "imprint" the ducklings in each group to either "same" objects or "different" objects, respectively. After a 30- 
min delay in darkness, the ducklings were tested for 10 min with two moving pairs of novel objects, one pair consisting of identical novel objects and the other pair of nonidentical novel objects. These researchers discovered that ducklings showed a preference to follow the novel-object pair that shared the same relationship (same or different) as their imprinted relationship between objects, which suggests that the concept of either same or different, respectively, was learned by each of the groups.

Our concept-learning research group recently studied the possibility that Clark's nutcrackers' predispositions for extensive caching of pine nuts might translate to more rapid same/different abstract-concept learning (Magnotti, Katz, Wright, \& Kelly, 2015; Wright, Magnotti, Katz, Leonard, \& Kelly, 2016). Cache location memory depends on complex relational processing of what (pine seeds), where (location), and when (cache locations change yearly). These components are closely related to episodic memory and involve abstract relational processing, similar to learning a samel different abstract concept. Therefore, we hypothesized that due to the nutcrackers' predisposition for caching and recovery of pine seeds, our group of wild-caught nutcrackers might have distinct advantages in learning the sameldifferent abstract concept relative to pigeons, and possibly relative even to monkeys. Our hypothesis was confirmed. Using the same task with the same procedures and stimuli, the nutcrackers as a group fully learned the samel different abstract concept with the same number of concept exemplars (128 set size) as monkeys, and they outperformed pigeons (256 set size). Moreover, the nutcrackers showed better-thanchance novel-stimulus transfer ( $66 \%$ correct) with the initial eight-picture training set, outperforming both monkeys and pigeons, which were both at 50\%, chance performance.

These findings were encouraging and appeared to support the role of nutcrackers' strong predisposition for food caching and retrieval in concept learning. To further examine this foodcaching hypothesis, we tested a cohort of ten black-billed magpies (Pica hudsonia) with the same procedures we had used to test nutcrackers (Wright et al., 2017). Magpies rely much less on cached foods than nutcrackers do, and are also more omnivorous and have better access to multiple food types throughout the year. If food-caching and retrieval were responsible for the nutcrackers' rapid, full concept learning, then magpies' full concept learning might turn out to be similar to that of pigeons. Alternatively, if skill in relational processing was built into the evolved neural architecture of these two corvid species, then the magpies' full concept learning should be equivalent to that of the nutcrackers (and primates). The findings supported the latter outcome: The two corvid species were virtually identical in their same/different concept learning; they attained full concept learning with the 128item set, equivalent to that of monkeys and surpassing that of pigeons. They also surpassed monkeys (and pigeons) in their partial concept learning with the initial eight-item set.

Skill in relational processing and same/different concept learning appear to be built into the evolved neural architecture of these two corvid species. Surprisingly, these bird brains - which are typically considered more primitive than primate brains - were actually competitive with, and even initially outperformed, what is typically considered a more elaborate brain in sameldifferent abstract-concept learning, a feat considered to represent advanced cognitive processing. The contrast in neural architectures between corvid and primate species is striking, but apparently environmental pressures for survival have selected and shaped these different neural architectures to successfully accomplish many of the same essential and intelligent behaviors (convergent evolution).

Other species, including ducks (Anas platyrhynchos domestica), experienced similar environmental pressures, raising the issue of how mature ducks might compare if they were tested in a same/different task similar to the one we used. Importantly, how might the rapid and impressive same or different concept learning from the imprinting task translate to flexible learning of a sameldifferent task like the one we used to test the other species? A well-established aspect of imprinting is that it is never forgotten and lasts a lifetime. Therefore, one possibility would be that mature ducks that had experienced same or different imprinting as per Martinho and Kacelnik (2016) might display enhanced samel different abstract-concept learning in a task similar to the one we used to test the other species, along with enhanced samel different abstract-concept learning relative to control groups imprinted on only single objects from the pairs used by Martinho and Kacelnik or to a control group imprinted on "mother duck." Another possible outcome might be that the imprinted ducks might display biases carried over from being imprinted on just same or just different; that is, they might respond disproportionately same or different, depending on their type of imprinting. Finally, imprinting of any type might have no effect on this sameldifferent abstract-concept learning and, similar to learning in the corvid species we tested, might depend more on flexible cognition from a neural architecture that had adapted (evolved) to environmental pressures to accomplish these essential and intelligent behaviors for survival.

\section{References}

Magnotti, J. F., Katz, J. S., Wright, A. A., \& Kelly, D. M. (2015). Superior abstract-concept learning by Clark's nutcrackers (Nucifraga columbiana). Biology Letters, 11, 20150148. doi:10.1098/rsbl. 2015.0148

Martinho, A., III, \& Kacelnik, A. (2016). Ducklings imprint on relational concept of "same or different.". Science, 353, 286-288.

Wright, A. A., \& Katz, J. S. (2006). Mechanisms of same/different concept learning in primates and avians. Behavioural Processes, 72, 234-254.

Wright, A. A., Magnotti, J. F., Katz, J. S., Leonard, K., \& Kelly, D. M. (2016). Concept learning set-size functions for Clark's nutcrackers. Journal of the Experimental Analysis of Behavior, 105, 76-84. doi: 10.1002/jeab. 174

Wright, A. A., Magnotti, J. F., Katz, J. S., Leonard, K., Vernouillet, A., \& Kelly, D. M. (2017). Corvids outperform pigeons and primates in learning a basic concept. Psychological Science. Advance online publication. doi:10.1177/0956797616685871 